\title{
Distributed Control for $\boldsymbol{n} \times \boldsymbol{n}$ Cooperative Systems Governed by Hyperbolic Operator of Infinite Order
}

\author{
A. H. Qamlo \\ Mathematical Sciences Department, Faculty of Applied Sciences, Umm AL-Qura University, Makkah, Saudi Arabia \\ Email: drahqamlo@yahoo.com
}

How to cite this paper: Qamlo, A.H. (2020) Distributed Control for $n \times n$ Cooperative Systems Governed by Hyperbolic Operator of Infinite Order. Advances in Pure Mathematics, 10, 728-738.

https://doi.org/10.4236/apm.2020.1012045

Received: November 20, 2020

Accepted: December 20, 2020

Published: December 23, 2020

Copyright $\odot 2020$ by author(s) and Scientific Research Publishing Inc. This work is licensed under the Creative Commons Attribution International License (CC BY 4.0).

http://creativecommons.org/licenses/by/4.0/

\begin{abstract}
In this study, a distributed optimal control problem for $n \times n$ cooperative hyperbolic systems with infinite order operators and Dirichlet conditions are considered. The existence and uniqueness of the state of these systems are proved. The necessary and sufficient conditions for optimality of distributed control with constraints are found, and the set of equations and inequalities that defining the optimal control of these systems is also obtained. Finally, some examples for the control problem without constraints are given.
\end{abstract}

\section{Keywords}

Cooperative Systems, Hyperbolic Systems, Optimal Control, Infinite Order, Distributed Control Problem, Dirichlet Conditions

\section{Introduction}

The earliest theory of optimal control was introduced by Lions [1].

Majority of the research in this field has focused on discussing the optimal control problem by using several operator types (such as elliptic, parabolic, or hyperbolic operators) [2] [3] [4].

The discussion was extended to systems involving different types of operators (such as infinite order [5]-[11] or infinite number of variables [12] [13] [14]).

In [3] [15] [16] [17], the studies continued to develop using different types of systems (cooperative or non-cooperative).

Based on the theories proposed by Lions [1] and Dubinskii [18] [19] [20], the distributed control problem with Dirichlet conditions for $2 \times 2$ non-cooperative hyperbolic systems involving infinite order operators was discussed in a previous study [17]; in this study, we extend this problem to $n \times n$ cooperative hyperbolic 
systems.

The system can be defined as

$$
\left.\begin{array}{l}
\frac{\partial^{2}}{\partial t^{2}} y_{i}+\sum_{|\alpha|=0}^{\infty}(-1)^{|\alpha|} a_{\alpha} D^{2 \alpha} y_{i}=\sum_{j=1}^{n} a_{i j} y_{j}+f_{i} \text { in } Q \\
y_{i} \rightarrow 0,|x| \rightarrow \infty \\
D^{\omega} y_{i}=0 \text { on } \Sigma \text { for }|\omega|=0,1,2, \cdots,|\omega| \leq \alpha-1, i=1,2, \cdots, n \\
y_{i}(x, 0)=y_{i, 0}(x), \frac{\partial y_{i}(x, 0)}{\partial t}=y_{i, 1}(x), x \in \Omega
\end{array}\right\}
$$

with $y_{i} \in L^{2}(Q), \frac{\partial y_{i}}{\partial t} \in L^{2}(Q)$.

Where

$a_{i j}>0$ for all $i \neq j$. (This implies that the system (1) is cooperative), (2)

$$
a_{i j}=a_{j i} \text { for all } 1 \leq i, j \leq n,
$$

and $Q=\Omega \times] 0, T[$ with boundary $\Sigma=\Gamma \times] 0, T[$.

This paper is constituted of four sections. Section 1 presents the Sobolev spaces of infinite order, which we refer to later in the paper. In section 2, the state of $n \times n$ cooperative system with Dirichlet conditions is studied. In Section 3, the formulation of the distributed control with constraints is introduced. Finally, Section 4 presents some examples for the control problem without constraints.

\section{Necessary Spaces: [18] [19] [20]}

The Sobolev spaces of infinite order operators, which are used in this study, have already been presented in Reference [17].

We will list them briefly below:

$$
\text { * } H^{\infty}(\Omega)=H^{\infty}\left\{a_{\alpha}, 2\right\}(\Omega)=\left\{\psi(x) \in C^{\infty}(\Omega): \sum_{|\alpha|=0}^{\infty} a_{\alpha}\left\|D^{\alpha} \psi\right\|_{2}^{2} \leq \infty\right\},
$$

* The conjugate space of $H^{\infty}(\Omega)$ is defined as,

$$
\text { * } H^{-\infty}(\Omega)=H^{-\infty}\left\{a_{\alpha}, 2\right\}(\Omega)=\left\{\vartheta(x): \vartheta(x)=\sum_{|\alpha|=0}^{\infty} a_{\alpha} D^{\alpha} \vartheta_{\alpha}(x)\right\},
$$

where $\vartheta_{\alpha} \in L^{2}(\Omega)$ and $\sum_{|\alpha|=0}^{\infty} a_{\alpha}\left\|D^{\alpha} \vartheta_{\alpha}\right\|_{2}^{2}<\infty$.

Then we have the following chains:

$$
\begin{aligned}
& * H^{\infty}(\Omega) \subseteq L^{2}(\Omega) \subseteq H^{-\infty}(\Omega), \\
& { }^{*} H_{0}^{\infty}(\Omega) \subseteq L^{2}(\Omega) \subseteq H_{0}^{-\infty}(\Omega),
\end{aligned}
$$

where $=\left\{\psi(x) \in C_{0}^{\infty}(\Omega):\|\psi\|^{2}=\sum_{|\alpha|=0}^{\infty} a_{\alpha}\left\|D^{\alpha} \psi\right\|_{2}^{2} \prec \infty,\left.D^{\omega} \psi\right|_{\Gamma}=0,|\omega| \leq \alpha\right\}$.

* $L^{2}(Q)=L^{2}\left(0, T, L^{2}(\Omega)\right)$ is a Hilbert space of measurable functions

$t \rightarrow \psi(t), t \in] 0, T\left[\right.$, that map an interval $(0, T)$ in to the space $L^{2}(\Omega)$, such

that: $\|\psi\|_{L^{2}(Q)}=\left(\int_{0}^{T}\|\psi(t)\|_{2}^{2} \mathrm{~d} t\right)^{\frac{1}{2}} \leq \infty$, and 


$$
(f, g)=\int_{0}^{T}(f(t), g(t))_{L^{2}(\Omega)} \mathrm{d} t,
$$

* In a similar manner as that of $L^{2}(Q)$, we obtain the constructed space $L^{2}\left(0, T, H_{0}^{\infty}(\Omega)\right)=L^{2}\left(H_{0}^{\infty}(Q)\right)$, and the following chains:

$$
\begin{gathered}
* L^{2}\left(H_{0}^{\infty}(Q)\right) \subseteq L^{2}(Q) \subseteq L^{2}\left(H_{0}^{-\infty}(Q)\right), \\
*\left(L^{2}\left(H_{0}^{\infty}(Q)\right)\right)^{n} \subseteq\left(L^{2}(Q)\right)^{n} \subseteq\left(L^{2}\left(H_{0}^{-\infty}(Q)\right)\right)^{n} .
\end{gathered}
$$

Finally,

$$
\text { * } W_{0}(0, T)=\left\{f \in L^{2}\left(H_{0}^{\infty}(Q)\right): \frac{\mathrm{d} f}{\mathrm{~d} t} \in L^{2}\left(H_{0}^{-\infty}(Q)\right)\right\},
$$

with the norm:

$$
\|f(t)\|_{W_{0}(0, T)}=\left(\int_{(0, T)}\|f(t)\|_{H_{0}^{\infty}(\Omega)}^{2} \mathrm{~d} t+\int_{(0, T)}\left\|\frac{\mathrm{d} f}{\mathrm{~d} t}\right\|_{H_{0}^{-\infty}(\Omega)}^{2} \mathrm{~d} t\right)^{1 / 2}
$$

which is also a Hilbert space.

\section{State of the System}

We study the following cooperative hyperbolic systems with Dirichlet conditions:

$$
\left.\begin{array}{l}
\frac{\partial^{2}}{\partial t^{2}} y_{i}+A y_{i}=\sum_{j=1}^{n} a_{i j} y_{j}+f_{i} \text { in } Q \\
y_{i} \rightarrow 0,|x| \rightarrow \infty \\
D^{\omega} y_{i}=0 \text { on } \Sigma \text { for }|\omega|=0,1,2, \cdots,|\omega| \leq \alpha-1, i=1,2, \cdots, n \\
y_{i}(x, 0)=y_{i, 0}(x), \frac{\partial y_{i}(x, 0)}{\partial t}=y_{i, 1}(x), x \in \Omega
\end{array}\right\}
$$

with $y_{i} \in L^{2}\left(H_{0}^{\infty}(Q)\right), \frac{\partial y_{i}}{\partial t} \in L_{2}(Q)$.

We have the operators $A \in \mathcal{L}\left(\left(L^{2}\left(H_{0}^{\infty}(Q)\right)\right)^{n},\left(L^{2}\left(H_{0}^{-\infty}(Q)\right)\right)^{n}\right)$ such that

$$
\begin{aligned}
& A\left(\bar{y}=\left(y_{1}, y_{2}, \cdots, y_{n}\right)\right)=\left(A y_{1}, A y_{2}, \cdots, A y_{n}\right) \\
& =\left(\sum_{|\alpha|=0}^{\infty}(-1)^{|\alpha|} a_{\alpha} D^{2 \alpha} y_{1}, \sum_{|\alpha|=0}^{\infty}(-1)^{|\alpha|} a_{\alpha} D^{2 \alpha} y_{2}, \cdots, \sum_{|\alpha|=0}^{\infty}(-1)^{|\alpha|} a_{\alpha} D^{2 \alpha} y_{n}\right),
\end{aligned}
$$

it is easy to write $A$ as a matrix take the form:

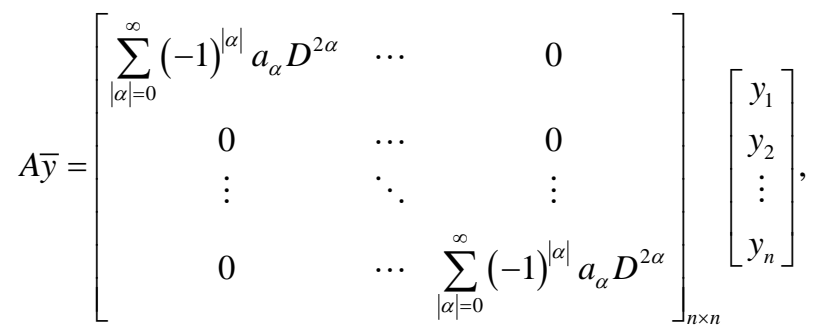


i.e.

$$
A y_{i}=\sum_{|\alpha|=0}^{\infty}(-1)^{|\alpha|} a_{\alpha} D^{2 \alpha} y_{i}, \quad i=1,2, \cdots, n
$$

Let $M$ be $(n \times n)$ square coefficients matrix such that

$$
\begin{aligned}
& M\left(\bar{y}=\left(y_{1}, y_{2}, \cdots, y_{n}\right)\right)=\left(\sum_{j=1}^{n} a_{1 j} y_{j}, \sum_{j=1}^{n} a_{2 j} y_{j}, \cdots, \sum_{j=1}^{n} a_{n j} y_{j}\right) \\
& =\left(a_{11} y_{1}+\cdots+a_{1 n} y_{n}, a_{21} y_{1}+\cdots+a_{2 n} y_{n}, a_{n 1} y_{1}+\cdots+a_{n n} y_{n}\right)
\end{aligned}
$$

i.e. $\quad M y=\sum_{j=1}^{n} a_{i j} y_{j}, i=1,2, \cdots, n$.

Let $S=A-M$, so that $S$ represents $(n \times n)$ square matrix takes the form

$$
S=\left[\begin{array}{cccc}
\sum_{|\alpha|=0}^{\infty}(-1)^{|\alpha|} a_{\alpha} D^{2 \alpha}-a_{11} & a_{12} & \cdots & a_{1 n} \\
a_{21} & \sum_{|\alpha|=0}^{\infty}(-1)^{|\alpha|} a_{\alpha} D^{2 \alpha}-a_{22} & \cdots & a_{2 n} \\
\vdots & \vdots & \ddots & \vdots \\
a_{n 1} & a_{n 2} & \cdots & \sum_{|\alpha|=0}^{\infty}(-1)^{|\alpha|} a_{\alpha} D^{2 \alpha}-a_{n n}
\end{array}\right]_{n \times n}
$$

Therefore, $S_{i} y_{i}=\sum_{|\alpha|=0}^{\infty}(-1)^{|\alpha|} a_{\alpha} D^{2 \alpha} y_{i}-\sum_{j=1}^{n} a_{i j} y_{j}, i=1,2, \cdots, n$.

Hence, we can rewrite the first equation in system (4) as follows:

$$
\frac{\partial^{2}}{\partial t^{2}} y_{i}+S_{i} y_{i}=f_{i} \text { in } Q
$$

Definition 1:

The bilinear form $\pi(t, \bar{y}, \bar{\phi})$ is defined on $\left(L^{2}\left(H_{0}^{\infty}(Q)\right)\right)^{n}$ as follows:

$$
\pi(t, \bar{y}, \bar{\phi})=(S y, \bar{\phi})_{\left(L^{2}(Q)\right)^{n}}, \bar{y}=\left(y_{i}\right)_{i=1}^{n}, \bar{\phi}=\left(\phi_{i}\right)_{i=1}^{n} \in\left(L^{2}\left(H_{0}^{\infty}(Q)\right)\right)^{n},
$$

where $S$ maps $\left(L^{2}\left(H_{0}^{\infty}(Q)\right)\right)^{n}$ onto $\left(L^{2}\left(H_{0}^{-\infty}(Q)\right)\right)^{n}$, so that

$$
\begin{gathered}
\pi(t, \bar{y}, \bar{\phi})=\sum_{i=1}^{n}\left(S_{i} y_{i}, \phi_{i}\right)_{L^{2}(Q)}, \\
\pi(t, \bar{y}, \bar{\phi})=\sum_{i=1}^{n}\left(\sum_{|\alpha|=0}^{\infty}(-1)^{|\alpha|} a_{\alpha} D^{2 \alpha} y_{i}-\sum_{j=1}^{n} a_{i j} y_{j}, \phi_{i}\right)_{L^{2}(Q)} \\
=\sum_{i=1}^{n} \int_{Q} \sum_{|\alpha|=0}^{\infty} a_{\alpha} D^{\alpha} y_{i} D^{\alpha} \phi_{i} \mathrm{~d} x \mathrm{~d} t-\sum_{i=1}^{n} \int_{Q} \sum_{j=1}^{n} a_{i j} y_{j} \phi_{i} \mathrm{~d} x \mathrm{~d} t .
\end{gathered}
$$

\section{Lemma 1:}

There exists a constant $c, c_{1} \in R$, such that:

$$
\pi(t, \bar{y}, \bar{y})+c_{1}\|y\|_{\left(L^{2}(Q)\right)^{n}}^{2} \geq c\|y\|_{\left(L^{2}\left(H_{0}^{\infty}(Q)\right)\right)^{n}}^{2}, c, c_{1}>0,
$$

that is, (6) is coercive on $\left(L^{2}\left(H_{0}^{\infty}(Q)\right)\right)^{n}$.

Proof:

We have:

$$
\pi(t, \bar{y}, \bar{\phi})=\sum_{i=1}^{n} \int_{Q} \sum_{|\alpha|=0}^{\infty} a_{\alpha} D^{\alpha} y_{i} D^{\alpha} \phi_{i} \mathrm{~d} x \mathrm{~d} t-\sum_{i=1}^{n} \int_{Q} \sum_{j=1}^{n} a_{i j} y_{j} \phi_{i} \mathrm{~d} x \mathrm{~d} t .
$$


Thus,

$$
\begin{aligned}
\pi(t, \bar{y}, \bar{y})= & \sum_{i=1}^{n} \int_{Q} \sum_{|\alpha|=0}^{\infty} a_{\alpha}\left|D^{\alpha} y_{i}\right|^{2} \mathrm{~d} x \mathrm{~d} t-\sum_{i=1}^{n} \int_{Q} a_{i i}\left|y_{i}\right|^{2} \mathrm{~d} x \mathrm{~d} t \\
& -\sum_{i \neq j}^{n} \int_{Q} a_{i j} y_{i} y_{j} \mathrm{~d} x \mathrm{~d} t
\end{aligned}
$$

then we deduce

$$
\begin{aligned}
& \pi(t, \bar{y}, \bar{y})+\sum_{i=1}^{n} \int_{Q} a_{i i}\left|y_{i}\right|^{2} \mathrm{~d} x \mathrm{~d} t+\sum_{i \neq j}^{n} \int_{Q} a_{i j} y_{i} y_{j} \mathrm{~d} x \mathrm{~d} t \\
& =\sum_{i=1}^{n} \int_{Q} \sum_{|\alpha|=0}^{\infty} a_{\alpha}\left|D^{\alpha} y_{i}\right|^{2} \mathrm{~d} x \mathrm{~d} t
\end{aligned}
$$

then,

$$
\pi(t, \bar{y}, \bar{y})+c_{1}\|y\|_{\left(L^{2}(Q)\right)^{n}}^{2} \geq\|y\|_{\left(L^{2}\left(H_{0}^{\infty}(Q)\right)\right)^{n}}^{2}, \quad c_{1}>0
$$

which proves the coerciveness condition on $\left(L^{2}\left(H_{0}^{\infty}(Q)\right)\right)^{n}$.

Lemma 2:

If (2), (3) and (7) are hold, then $\exists ! \bar{y}=\left(y_{i}\right)_{i=1}^{n} \in\left(L^{2}\left(H_{0}^{\infty}(Q)\right)\right)^{n}$ for system (4), for $f_{i}=f_{i}(x, t) \in L^{2}(Q)$.

\section{Proof:}

Let $\bar{\psi}=\left(\psi_{i}\right)_{i=1}^{n} \rightarrow L(\bar{\psi})$ be a continuous linear form defined on $\left(L^{2}\left(H_{0}^{\infty}(Q)\right)\right)^{n}$ by

$$
\begin{gathered}
\forall \bar{\psi}=\left(\psi_{i}\right)_{i=1}^{n} \in\left(L^{2}\left(H_{0}^{\infty}(Q)\right)\right)^{n}, \\
L(\bar{\psi})=\sum_{i=1}^{n}\left\{\int_{Q} f_{i} \psi_{i} \mathrm{~d} x \mathrm{~d} t+\int_{\Omega} y_{i, 1} \psi_{i}(x, 0) \mathrm{d} x\right\},
\end{gathered}
$$

where $f_{i} \in L^{2}(Q), y_{i, 0} \in L^{2}(\Omega)$ and $y_{i, 1} \in L^{2}(\Omega)$.

Then, by the Lax-Milgram lemma,

$\exists ! \bar{y} \in\left(L^{2}\left(H_{0}^{\infty}(Q)\right)\right)^{n}$ such that

$$
L(\bar{\psi})=\frac{\partial^{2}}{\partial t^{2}}\left(y_{i}, \psi_{i}\right)+\pi(t, \bar{y}, \bar{\psi}), \forall \bar{\psi}=\left(\psi_{i}\right)_{i=1}^{n} \in\left(L^{2}\left(H_{0}^{\infty}(Q)\right)\right)^{n} .
$$

Now, let us multiply system (4) by $\psi_{i}$, and then integrate it over $Q$ :

$$
\sum_{i=1}^{n} \int_{Q}\left(\frac{\partial^{2}}{\partial t^{2}} y_{i}+A y_{i}\right) \psi_{i} \mathrm{~d} x \mathrm{~d} t-\sum_{j=1}^{n} \int_{Q} a_{i j} y_{j} \psi_{i} \mathrm{~d} x \mathrm{~d} t=\sum_{i=1}^{n} \int_{Q} f_{i} \psi_{i} \mathrm{~d} x \mathrm{~d} t .
$$

By using Green's formula:

$$
\begin{aligned}
& \sum_{i=1}^{n}\left\{\int_{Q} \frac{\partial^{2} \psi_{i}}{\partial t^{2}} y_{i} \mathrm{~d} x \mathrm{~d} t+\int_{Q} \sum_{|\alpha|=0}^{\infty} a_{\alpha} D^{\alpha} y_{i} D^{\alpha} \psi_{i} \mathrm{~d} x \mathrm{~d} t-\sum_{j=1}^{n} \int_{Q} a_{i j} y_{j} \psi_{i} \mathrm{~d} x \mathrm{~d} t\right. \\
& \left.+\int_{\Omega} \frac{\partial y_{i}(x, 0)}{\partial t} \psi_{i}(x, 0) \mathrm{d} x-\int_{\Sigma} \frac{\partial y_{i}}{\partial v_{A}} \psi_{i} \mathrm{~d} \Sigma\right\}=\sum_{i=1}^{n} \int_{Q} f_{i} \psi_{i} \mathrm{~d} x \mathrm{~d} t
\end{aligned}
$$

from (6), (8) and (9) we have

$$
\sum_{i=1}^{n}\left\{\int_{\Omega} \frac{\partial y_{i}(x, 0)}{\partial t} \psi_{i}(x, 0) \mathrm{d} x-\int_{\Sigma} \frac{\partial y_{i}}{\partial v_{A}} \psi_{i} \mathrm{~d} \Sigma\right\}=\sum_{i=1}^{n} \int_{Q} y_{i, 1}(x) \psi_{i}(x, 0) \mathrm{d} x
$$

Then, we deduce that 


$$
\begin{gathered}
D^{\omega} y_{i}=0 \text { on } \Sigma \text { for }|\omega|=0,1,2,3, \cdots,|\omega| \leq \alpha-1, i=1,2,3, \cdots, n \\
\frac{\partial y_{i}(x, 0)}{\partial t}=y_{i, 1}(x), \quad x \in \Omega,
\end{gathered}
$$

Thus, the proof is complete.

\section{Control Problem with Constraints}

The space $U=\left(L^{2}(Q)\right)^{n}$ is the space of controls $\bar{u}=\left(u_{i}\right)_{i=1}^{n}$.

The state of the system $\bar{y}(\bar{u})=\left(y_{i}(\bar{u})\right)_{i=1}^{n} \in\left(L^{2}\left(H^{\infty}(Q)\right)\right)^{n}$ is determined by the solution of

$$
\left.\begin{array}{ll} 
& \frac{\partial^{2}}{\partial t^{2}} y_{i}(\bar{u})+A y_{i}(\bar{u})=\sum_{j=1}^{n} a_{i j} y_{j}+f_{i}+u_{i} \text { in } Q, \\
& y_{i} \rightarrow 0,|x| \rightarrow \infty, \\
& D^{\omega} y_{i}=0 \text { on } \Sigma \text { for }|\omega|=0,1,2,3, \cdots,|\omega| \leq \alpha-1, i=1,2,3, \cdots, n \\
& y_{i}(x, 0, \bar{u})=y_{i, 0}(x), \frac{\partial y_{i}(x, 0, \bar{u})}{\partial t}=y_{i, 1}(x), x \in \Omega, \\
\text { with } \quad y_{i} \in L^{2}\left(H_{0}^{\infty}(Q)\right), \frac{\partial y_{i}}{\partial t} \in L_{2}(Q) .
\end{array}\right\}
$$

The observation function is given by

$$
\bar{z}(\bar{u})=\left(z_{i}(\bar{u})\right)_{i=1}^{n}=\left(y_{i}(\bar{u})\right)_{i=1}^{n}=\bar{y}(\bar{u}) .
$$

The cost function $J(\bar{u})$ is given by

$$
J(\bar{u})=\sum_{i=1}^{n}\left\|y_{i}(\bar{u})-z_{i d}\right\|_{L^{2}(Q)}^{2}+M \sum_{i=1}^{n}\left\|u_{i}\right\|_{L^{2}(Q)}^{2}
$$

where $\bar{z}_{d}=\left(z_{i d}\right)_{i=1}^{n} \in\left(L^{2}(Q)\right)^{n}$ and $M \geq 0$ is a constant.

Then, the control problem is to minimize $J$ over $U_{a d}$ which is a closed convex subset of $U=\left(L^{2}(Q)\right)^{n}$.

i.e. to determine $\bar{u}$ such that

$$
J(\bar{u})=\inf _{\bar{v} \in U_{a d}} J(\bar{v}), \quad \bar{v}=\left(v_{i}\right)_{i=1}^{n} .
$$

Based on the above data and previous results, we have the following theorem:

\section{Theorem 1:}

Assuming that (7),(10) and (11) hold, $\exists$ ! the optimal control $\bar{u}=\left(u_{i}\right)_{i=1}^{n} \in U_{a d}$ such that: $J(\bar{u}) \leq J(\bar{v}), \forall \bar{v}=\left(v_{i}\right)_{i=1}^{n} \in U_{a d}$, and it is determined by:

$$
\left.\begin{array}{l}
\frac{\partial^{2} p_{i}(\bar{u})}{\partial t^{2}}+A p_{i}(\bar{u})-\sum_{j=1}^{n} a_{i j} p_{j}=y_{i}(\bar{u})-z_{i d} \text { in } Q \\
D^{\omega} p_{i}(\bar{u})=0 \text { on } \Sigma \text { for }|\omega|=0,1,2, \cdots,|\omega| \leq \alpha-1, i=1,2, \cdots, n \\
p_{i}(x, 0, \bar{u})=0, \frac{\partial p_{i}(x, 0, \bar{u})}{\partial t}=0, x \in \Omega
\end{array}\right\}
$$

with $y_{i}, p_{i} \in L^{2}\left(H_{0}^{\infty}(Q)\right), \frac{\partial y_{i}}{\partial t}, \frac{\partial p_{i}}{\partial t} \in L_{2}(Q)$ 
and

$$
\sum_{i=1}^{n}\left(p_{i}(\bar{u})+M u_{i}, v_{i}-u_{i}\right)_{L^{2}(Q)} \geq 0, \forall \bar{v}=\left(v_{i}\right)_{i=1}^{n} \in U_{a d}
$$

where $p_{i}(\bar{u})$ is the adjoint state.

Proof:

As in [1], $\bar{u}=\left(u_{i}\right)_{i=1}^{n} \in U_{a d}$ is determined by:

$$
\sum_{i=1}^{n} J_{i}^{\prime}(\bar{u})\left(v_{i}-u_{i}\right) \geq 0, \forall \bar{v}=\left(v_{i}\right)_{i=1}^{n} \in U_{a d},
$$

i.e.

$$
\sum_{i=1}^{n}\left(y_{i}(\bar{u})-z_{i d}, y_{i}(\bar{v})-y_{i}(\bar{u})\right)_{L^{2}(Q)}+\sum_{i=1}^{n} M\left(u_{i}, v_{i}-u_{i}\right)_{L^{2}(Q)} \geq 0
$$

which is equivalent to:

$$
\sum_{i=1}^{n} \int_{0}^{T}\left(y_{i}(\bar{u})-z_{i d}, y_{i}(\bar{v})-y_{i}(\bar{u})\right)_{L^{2}(\Omega)} \mathrm{d} t+\sum_{i=1}^{n} M\left(u_{i}, v_{i}-u_{i}\right)_{L^{2}(Q)} \geq 0 .
$$

Now, let us define a hyperbolic infinite order operator $B$ as follows:

$$
B \bar{y}(\bar{u})=B y_{i}(\bar{u})=\frac{\partial^{2} y_{i}(\bar{u})}{\partial t^{2}}+A y_{i}(\bar{u})-\sum_{j=1}^{n} a_{i j} y_{j}, \forall \bar{u}=\left(u_{i}\right)_{i=1}^{n} \in U_{a d},
$$

Since, $(\bar{p}, B \bar{y})_{L^{2}(Q)}=\int_{0}^{T}\left(p_{i}(\bar{u}), \frac{\partial^{2} y_{i}(\bar{u})}{\partial t^{2}}+A y_{i}(\bar{u})-\sum_{j=1}^{n} a_{i j} y_{j}\right)_{L^{2}(\Omega)} \mathrm{d} t$, from (3), we obtain

$$
\begin{aligned}
(\bar{p}, B \bar{y})_{L^{2}(Q)} & =\int_{0}^{T}\left(\frac{\partial^{2} p_{i}(\bar{u})}{\partial t^{2}}+A p_{i}(\bar{u})-\sum_{j=1}^{n} a_{i j} p_{j}, y_{i}(\bar{u})\right)_{L^{2}(\Omega)} \mathrm{d} t \\
& =\left(B^{*} \bar{p}, \bar{y}\right)_{L^{2}(Q)},
\end{aligned}
$$

then $B^{*} \bar{p}(\bar{u})=B^{*}\left(p_{i}(\bar{u})\right)=\left(\frac{\partial^{2} p_{i}(\bar{u})}{\partial t^{2}}+A p_{i}(\bar{u})-\sum_{j=1}^{n} a_{i j} p_{j}\right), i=1,2, \cdots, n$.

Now, let us set the following notation:

$$
S^{*} \bar{p}(\bar{u})=S_{i}^{*}\left(p_{i}(\bar{u})\right)=A p_{i}(\bar{u})-\sum_{j=1}^{n} a_{i j} p_{j}, i=1,2, \cdots, n .
$$

According to the form of the adjoint equation in [1]:

$$
\frac{\partial^{2} p(\bar{u})}{\partial t^{2}}+S_{i}^{*} p(\bar{u})=y(\bar{u})-z_{d},
$$

and by Lemma 2,

$\exists$ ! Solution $p_{i}(\bar{u}) \in L^{2}(Q)$ for (12).

Now, we transform (14) as follows:

we multiply (12) by $\left(y_{i}(\bar{v})-y_{i}(\bar{u})\right)$ and integrating between $0, T$, then we obtain:

$$
\begin{aligned}
& \int_{0}^{T}\left(y_{i}(\bar{u})-z_{i d}, y_{i}(\bar{v})-y_{i}(\bar{u})\right)_{L^{2}(\Omega)} \mathrm{d} t \\
& =\int_{0}^{T}\left(\left(\frac{\partial^{2}}{\partial t^{2}}+A\right) p_{i}(\bar{u})-\sum_{j=1}^{n} a_{i j} p_{j},\left(y_{i}(\bar{v})-y_{i}(\bar{u})\right)\right)_{L^{2}(\Omega)} \mathrm{d} t
\end{aligned}
$$




$$
\begin{aligned}
& =\int_{0}^{T}\left(p_{i}(\bar{u}),\left(\frac{\partial^{2}}{\partial t^{2}}+A\right)\left(y_{i}(\bar{v})-y_{i}(\bar{u})-\sum_{j=1}^{n} a_{i j}\left(y_{i}(\bar{v})-y_{i}(\bar{u})\right)\right)\right)_{L^{2}(\Omega)} \mathrm{d} t \\
& =\int_{0}^{T}\left(p_{i}(\bar{u}), v_{i}-u_{i}\right)_{L^{2}(\Omega)} \mathrm{d} t,
\end{aligned}
$$

hence (14) becomes

$$
\sum_{i=1}^{n} \int_{0}^{T}\left(p_{i}(\bar{u}), v_{i}-u_{i}\right)_{L^{2}(\Omega)} \mathrm{d} t+\sum_{i=1}^{n} M\left(u_{i}, v_{i}-u_{i}\right)_{L^{2}(Q)} \geq 0, \quad \forall \bar{v}=\left(v_{i}\right)_{i=1}^{n} \in U_{a d},
$$

i.e. $\sum_{i=1}^{n} \int_{0}^{T}\left(p_{i}(\bar{u})+M u_{i}, v_{i}-u_{i}\right)_{L^{2}(\Omega)} \mathrm{d} t \geq 0, \forall \bar{v}=\left(v_{i}\right)_{i=1}^{n} \in U_{a d}$.

Thus, the proof is complete.

\section{Control Problem without Constraints}

1) The case if $U_{a d}=\left(L^{2}(Q)\right)^{n}$ i.e. (there are no constraints on the control $\bar{u})$, then (13) takes the form $p_{i}(\bar{u})+N_{i} u_{i}=0, x \in Q$, hence

$$
u_{i}=-N_{i}^{-1} p_{i}(\bar{u}) \text {. }
$$

\section{Example 1:}

Let us consider $n=2$ in (1), also (2) and (3) are satisfied, the space $\left(L_{2}(Q)\right)^{2}$ is the space of controls $u=\left(u_{1}, u_{2}\right)$ and the state

$$
\begin{aligned}
y(u)= & \left(y_{1}(u), y_{2}(u)\right) \in\left(L^{2}\left(H_{0}^{\infty}(Q)\right)\right)^{2} \text { is determined by: } \\
& \left\{\begin{array}{l}
\frac{\partial^{2} y_{1}(\bar{u})}{\partial t^{2}}+A y_{1}(\bar{u})+N_{1}^{-1} p_{1}(\bar{u})=a_{11} y_{1}(\bar{u})+a_{12} y_{2}(\bar{u})+f_{1} \text { in } Q, \\
\frac{\partial^{2} y_{2}(\bar{u})}{\partial t^{2}}+A y_{2}(\bar{u})+N_{2}^{-1} p_{2}(\bar{u})=a_{21} y_{1}(\bar{u})+a_{22} y_{2}(\bar{u})+f_{2} \text { in } Q, \\
y_{1}, y_{2} \rightarrow 0,|x| \rightarrow \infty, \\
\left.y_{1}(\bar{u})\right|_{\Sigma}=0,\left.\quad y_{2}(\bar{u})\right|_{\Sigma}=0, \\
y_{1}(x, 0 ; \bar{u})=y_{0,1}(x), \frac{\partial y_{1}(x, 0 ; \bar{u})}{\partial t}=y_{1,1}(x), \quad x \in \Omega, \\
y_{2}(x, 0 ; \bar{u})=y_{0,2}(x), \frac{\partial y_{2}(x, 0 ; \bar{u})}{\partial t}=y_{1,2}(x), \quad x \in \Omega .
\end{array}\right. \\
& \left\{\begin{array}{l}
y_{1}(\bar{u}), y_{2}(\bar{u}), \frac{\partial y_{1}(\bar{u})}{\partial t}, \frac{\partial y_{2}(\bar{u})}{\partial t} \in L^{2}(Q) . \\
\frac{\partial^{2} p_{1}(\bar{u})}{\partial t^{2}}+A p_{1}(\bar{u})-a_{11} p_{1}(\bar{u})-a_{12} p_{2}(\bar{u})=y_{1}(\bar{u})-z_{d 1} \text { in } Q, \\
\frac{\partial^{2} p_{2}(\bar{u})}{\partial t^{2}}+A p_{2}(\bar{u})-a_{21} p_{1}(\bar{u})-a_{22} p_{2}(\bar{u})=y_{2}(\bar{u})-z_{d 2} \text { in } Q, \\
\left.p_{1}(\bar{u})\right|_{\Sigma}=0,\left.p_{2}(\bar{u})\right|_{\Sigma}=0, \\
p_{1}(x, T, \bar{u})=0, \frac{\partial p_{1}(x, T u)}{\partial t}=0, x \in \Omega, \\
p_{2}(x, T, \bar{u})=0, \frac{\partial p_{2}(x, T u)}{\partial t}=0, x \in \Omega,
\end{array}, \frac{\partial y_{2}(\bar{u})}{\partial t} \in L^{2}(Q), p_{1}, p_{2}, \frac{\partial p_{1}(\bar{u})}{\partial t}, \frac{\partial p_{2}(\bar{u})}{\partial t} \in L^{2}(Q),\right.
\end{aligned}
$$




$$
u_{1}=-N_{1}^{-1} p_{1}(\bar{u}), u_{2}=-N_{2}^{-1} p_{2}(\bar{u}), \forall \bar{u}=\left(u_{1}, u_{2}\right) \in U_{a d},
$$

together with (16), where $p(\bar{u})=\left(p_{1}(\bar{u}), p_{2}(\bar{u})\right)$ is the adjoint state.

2) The case if there are no constraints on $u_{1}$,

i.e. $U_{a d}=\left\{\bar{u}: u_{1}\right.$ arbitrary in $L^{2}(Q), u_{i} \geq 0$ a.e. in $\left.Q, i=2, \cdots, n\right\}$,

hence, (13) takes the following form:

$$
\left\{\begin{array}{l}
p_{1}(\bar{u})+N_{1} u_{1}=0, \\
p_{i}(\bar{u})+N_{i} u_{i} \geq 0, u_{i} \geq 0 \text { a.e. } i=2, \cdots, n, \\
u_{i}\left(p_{i}(\bar{u})+N_{i} u_{i}\right)=0, i=2, \cdots, n .
\end{array}\right.
$$

\section{Example 2:}

If we take $n=2$,

$$
\text { then } U_{a d}=\left\{\bar{u} / u_{1} \text { arbitrary in } L^{2}(Q), u_{2} \geq 0 \text { a.e. in } Q\right\} \text {. }
$$

So, (13) is equivalent to

$$
\left\{\begin{array}{l}
p_{1}(\bar{u})+N_{1} u_{1}=0, \\
p_{2}(\bar{u})+N_{2} u_{2} \geq 0, u_{2} \geq 0 \text { a.e. } \\
u_{2}\left(p_{2}(\bar{u})+N_{2} u_{2}\right)=0 .
\end{array}\right.
$$

so, the optimal control is determined by:

$$
\begin{aligned}
& \int \frac{\partial^{2} y_{1}(\bar{u})}{\partial t^{2}}+A y_{1}(\bar{u})-a_{11} y_{1}(\bar{u})-a_{12} y_{2}(\bar{u})+N_{1}^{-1} p_{1}(\bar{u})=f_{1} \text { in } Q, \\
& \frac{\partial^{2} y_{2}(\bar{u})}{\partial t^{2}}+A y_{2}(\bar{u})-a_{21} y_{1}(\bar{u})-a_{22} y_{2}(\bar{u})-f_{2} \geq 0 \text { in } Q \text {, } \\
& \frac{\partial^{2} p_{1}(\bar{u})}{\partial t^{2}}+A p_{1}(\bar{u})-a_{11} p_{1}(\bar{u})-a_{12} p_{2}(\bar{u})=y_{1}(\bar{u})-z_{d 1} \text { in } Q, \\
& \frac{\partial^{2} p_{2}(\bar{u})}{\partial t^{2}}+A p_{2}(\bar{u})-a_{21} p_{1}(\bar{u})-a_{22} p_{2}(\bar{u})=y_{2}(\bar{u})-z_{d 2} \text { in } Q, \\
& p_{2}(\bar{u})+N_{2}\left\{\frac{\partial^{2} y_{2}(\bar{u})}{\partial t^{2}}+A y_{2}(\bar{u})-a_{21} y_{1}(\bar{u})-a_{22} y_{2}(\bar{u})-f_{2}\right\} \geq 0, \\
& \left\{\left(\frac{\partial^{2} y_{2}(\bar{u})}{\partial t^{2}}+A y_{2}(\bar{u})-a_{21} y_{1}(\bar{u})-a_{22} y_{2}(\bar{u})-f_{2}\right)\right. \\
& \cdot\left(p_{2}(\bar{u})+N_{2}\left(\frac{\partial^{2} y_{2}(\bar{u})}{\partial t^{2}}+A y_{2}(\bar{u})-a_{21} y_{1}(\bar{u})-a_{22} y_{2}(u)-f_{2}\right)\right)=0 \text {, } \\
& \left.y_{1}(\bar{u})\right|_{\Sigma}=0,\left.\quad y_{2}(\bar{u})\right|_{\Sigma}=0,\left.\quad p_{1}(\bar{u})\right|_{\Sigma}=0,\left.\quad p_{2}(\bar{u})\right|_{\Sigma}=0, \\
& y_{1}(x, 0 ; \bar{u})=y_{0,1}(x), \frac{\partial y_{1}(x, 0 ; \bar{u})}{\partial t}=y_{1,1}(x), x \in \Omega \text {, } \\
& y_{2}(x, 0 ; \bar{u})=y_{0,2}(x), \frac{\partial y_{2}(x, 0 ; \bar{u})}{\partial t}=y_{1,2}(x), x \in \Omega \text {, } \\
& p_{1}(x, T, \bar{u})=p_{2}(x, T, \bar{u})=0, x \in \Omega, \\
& \frac{\partial p_{1}(x, T u)}{\partial t}=\frac{\partial p_{2}(x, T u)}{\partial t}=0, x \in \Omega \text {. }
\end{aligned}
$$


Further

$$
\left\{\begin{array}{l}
u_{1}=N_{1}^{-1} p_{1}(\bar{u}), \\
u_{2}=\frac{\partial^{2} y_{2}(\bar{u})}{\partial t^{2}}+A y_{2}(\bar{u})-a_{21} y_{1}(\bar{u})-a_{22} y_{2}(\bar{u})-f_{2} .
\end{array}\right.
$$

\section{Conclusion}

In this paper, we have some important results. First of all we proved the existence and uniqueness of the state for system (4), which is $(2 \times 2)$ cooperative hyperbolic systems involving infinite order operators (Lemma 2). Then we found the necessary and sufficient conditions of optimality for system (10), that give the characterization of optimal control (Theorem 1).

Finally, we derived the necessary and sufficient conditions of optimality for some cases without control constraints.

Also it is evident that by modifying:

- the nature of the control (distributed, boundary),

- the nature of the observation (distributed, boundary),

- the initial differential system,

- the type of equation (elliptic, parabolic and hyperbolic),

- the type of system (non-cooperative, cooperative),

- the order of equation, many of variations on the above problem are possible to study with the help of Lions formalism.

\section{Acknowledgements}

The authors thank the anonymous referees for their valuable suggestions which led to the improvement of the manuscript.

\section{Conflicts of Interest}

The author declares no conflicts of interest regarding the publication of this paper.

\section{References}

[1] Lions, J.L. (1971) Optimal Control of Systems Governed by Partial Differential Equations. Vol. 170, Springer Verlag, Berlin, Heidelberg.

[2] Qamlo, A.H. (2008) Boundary Control for Cooperative Systems Involving Parabolic Operators with an Infinite Number of Variables. Advances in Differential Equations and Control Processes, 2, 135-151.

[3] Qamlo, A.H. (2014) An Optimization Problem of Boundary Type for Cooperative Hyperbolic Systems Involving Schrödinger Operator. Intelligent Control and $\mathrm{Au}$ tomation, 5, 262-271. http://dx.doi.org/10.4236/ica.2014.54028

[4] Serag, H.M. and Qamlo, A.H. (2005) On Elliptic Systems Involving Schrödinger Operators. The Mediterranean Journal of Measurement and Control, 1, 91-96.

[5] Bahaa, G.M. and Qamlo, A.H. (2013) Boundary Control Problem for Infinite Order Parabolic System with Time Delay and Control Constraints. European Journal of Scientific Research, 104, 392-406. 
[6] Bahaa, G.M. and El-Gohany, E. (2013) Optimal Control Problem for $(\mathrm{n} \times \mathrm{n})$ Infinite Order Parabolic Systems. International Journal of Applied Mathematics \& Statistics, 36, 26-41.

[7] El-Saify, H.A. (2006) Optimality Conditions for Infinite Order Hyperbolic Differential System. Journal of Dynamical and Control Systems, 12, 313-330. https://doi.org/10.1007/s10450-006-0002-1

[8] Gali, I.M. (1982) Optimal Control of Systems Governed by Elliptic Operator of Infinite Order. In: Everitt, W. and Sleeman, B., Eds., Ordinary and Partial Differential Equations. Lecture Notes in Mathematics, Vol. 964, Springer, Berlin, Heidelberg, 263-271. https://doi.org/10.1007/BFb006500

[9] Gali, I.M. and El-Saify, H.A. (1983) Control of System Governed by Infinite Order Equation of Hyperbolic Type. Proceeding of the International Conference on Functional-Differential Systems and Related Topics, Vol. 3, Poland, 99-103.

[10] Gali, I.M., El-Saify, H.A. and El-Zahaby, S.A. (1983) Distributed Control of a System Governed by Dirichlet and Neumann Problems for Elliptic Equations of Infinite Order. Proceeding of the International Conference on Functional-Differential Systems and Related Topics, Vol. 3, Poland, 83-87.

[11] Kotarski, W. and Bahaa, G.M. (2005) Optimal Control Problem for Infinite Order Hyperbolic System with Mixed Control-State Constraints. European Journal of Control, 11,150-156. https://doi.org/10.3166/ejc.11.150-156

[12] Kotarski, W., El-Saify, H.A. and Shehata, M.A. (2007) Time Optimal Control of Parabolic Lag Systems with an Infinite Number of Variables. Journal of the Egyptian Mathematical Society, 15, 21-34.

[13] Qamlo, A.H. (2009) Distributed Control of Cooperative Systems Involving Hyperbolic Operators with an Infinite Number of Variables. International Journal of Functional Analysis, Operator Theory and Applications, 1, 115-128.

[14] Serag, H.M. (2007) Distributed Control for Cooperative Systems Involving Parabolic Operators with an Infinite Number of Variables. IMA Journal of Mathematical Control and Information, 24, 149-161. https://doi.org/10.1093/imamci/dnl018

[15] Qamlo, A.H. (2013) Distributed Control for Cooperative Hyperbolic Systems Involving Schrödinger Operator. International Journal of Dynamics and Control, 1, 54-59. https://doi.org/10.1007/s40435-013-0007-Z

[16] Qamlo, A.H., Serag, H.M. and Al-zahrani, E. (2015) Optimal Control for Non-Cooperative Parabolic Systems with Conjugation Conditions. European Journal of Scientific Research, 131, 215-226.

[17] Qamlo, A.H. and Bedaiwi, G.M. (2017) Distributed Control for $2 \times 2$ Coupled Infinite Order Hyperbolic Systems. Advances in Differential Equations and Control Processes, 18, 201-227. http://dx.doi.org/10.17654/DE018040201

[18] Dubinskii, J.A. (1976) Non-Triviality of Sobolev Spaces of Infinite Order for a Full Euclidean Space and a Torus. Mathematics of the USSR-Sbornik, 29, 393-401. https://doi.org/10.1070/SM1976v029n03ABEH003675

[19] Dubinskii, J.A. (1981) About One Method for Solving Partial Differential Equations. Doklady Akademii Nauk SSSR, 258, 780-784.

[20] Dubinskii, J.A. (1986) Sobolev Spaces in Infinite Order and Differential Equations. Vol. 3, Springer, Netherland, 1-157. 